\title{
Wernicke's Encephalopathy after Sleeve Gastrectomy for Morbid Obesity - A Case Report -
}

\author{
Hyo Jun Jeong, M.D., Ji Woong Park, M.D., Yong Jin Kim, M.D. ', \\ Yang Gyun Lee, M.D., Yi Wook Jang, M.D., Jun Won Seo, M.D.
}

Departments of Physical and Rehabilitation Medicine and ${ }^{1}$ General Surgery, Soonchunhyang University Hospital, Soonchunhyang University College of Medicine, Seoul 140-743, Korea

\begin{abstract}
Morbid obesity is a curable systemic disease that can cause several complications, including hypertension, diabetes mellitus, and osteoarthritis. However, it is not easy to control solely by conservative management. Bariatric surgeries, such as sleeve gastrectomy and gastric banding, are recently developed treatments that are applied to patients with morbid obesity in Korea. However, gastric surgery can cause surgical or metabolic complications, such as thiamine deficiency, which can lead to Wernicke's encephalopathy. This metabolic complication presents with typical symptoms of confusion, ophthalmoplegia, nystagmus, and ataxia. In this case report, we present a case of Wernicke's encephalopathy, which developed slowly following sleeve gastrectomy in a patient with morbid obesity.
\end{abstract}

Key Words Wernicke's encephalopathy, Sleeve gastrectomy, Morbid obesity

\section{INTRODUCTION}

Morbid obesity is defined as a body mass index of over $40 \mathrm{~kg} / \mathrm{cm}^{2}$, which worsens a patient's quality of life due to complications such as hypertension, diabetes mellitus, and osteoarthritis. The prevalence of morbid obesity is currently increasing in Korea. It is very difficult to achieve a curative effect in morbid obesity with only conservative

Received August 19, 2010; Accepted December 13, 2010

Corresponding author: Ji Woong Park

Department of Physical and Rehabilitation Medicine, Soonchunhyang University Hospital, Soonchunhyang University College of Medicine, 22, Daesagwan-gil, Yongsan-gu, Seoul 140-743, Korea

Tel: +82-2-709-9370, Fax: +82-2-709-9265, E-mail: spotdoc88@gmail.com (c) This is an open-access article distributed under the terms of the Creative Commons Attribution Non-Commercial License (http://creativecommons.org/ licenses/by-nc/3.0) which permits unrestricted noncommercial use, distribution, and reproduction in any medium, provided the original work is properly cited.

Copyright $\odot 2011$ by Korean Academy of Rehabilitation Medicine treatment. Therefore, surgical treatments have recently been recommended. Surgical treatments for morbid obesity are called bariatric surgery and typically include bypass surgery, gastric banding, and sleeve gastrectomy, which are performed according to their indications. However, even though such surgical treatments are effective for body weight reduction, they may cause complications such as symptomatic anastomotic leakage, infection, esophageal motility disorder, malabsorption, or metabolic disorder. ${ }^{1}$ In other studies, additional complications have been reported that include various aspects of Wernicke's encephalopathy, which occurs due to thiamin deficiency after bariatric surgeries such as sleeve gastrectomy for morbid obesity. ${ }^{2-4}$ However, bariatric surgery has not been widely popular domestically until recently, and there are no relevant reports on complications or medical treatments. In this 
paper, we report on a patient diagnosed with Wernicke's encephalopathy, which occurred after sleeve surgery.

\section{CASE REPORT}

The patient was a 24-year-old male who experienced difficulty in walking for three months prior to our examination, and complained of dysarthria as well as diplopia. In his personal history, he had undergone laparoscopic sleeve gastrectomy surgery in the depart ment of surgery seven months prior to our examination. At that time, he was in a state of morbid obesity, and he was $190 \mathrm{~cm}$ tall, weighed $170 \mathrm{~kg}$, and had a $47.1 \mathrm{~kg} / \mathrm{cm}^{2}$ body mass index (BMI). The patient had hypertension and diabetes mellitus for one year before surgery, but no medical history of alcohol addiction, hepatitis, or tuberculosis. After the initial surgery, he had to undergo additional surgery twice due to leakage of gastroesophageal anastomosis. However, the leakage was continuous, so total parenteral nutrition was given for approximately 60 days. Stent insertion was then performed to begin parenteral nutrition. Subsequently, the patient exhibited a confused mental state and symptoms of delirium. The severity of the symptoms was weak, and his general condition gradually improved even with conservative treatment. The symptoms of delirium were considered temporary symptoms that often appear after an operation. Therefore, the patient left the hospital.

In the physical examination that was performed when the patient was admitted to the hospital, he presented with alert consciousness without a sign of cognitive deficiency. At that time, his weight was $110 \mathrm{~kg}$, which was $60 \mathrm{~kg}$ lighter than before the operation, and his BMI was $30.5 \mathrm{~kg} / \mathrm{cm}^{2}$. Judging from these data, his morbid obesity was remarkably improved. Sensory examination was normal both for superficial and deep sensation. However, as a result of a Medical Research Council of Great Britain (MRC) examination, it was found that the muscle strength of both arms and legs appeared to be at a level of $4-4+/ 5$, showing slight muscle weakness. Dysmetria was observed in both hands and feet, which was more serious on the left side of his body compared to the right side. It was impossible for the patient to sit down, stand, or walk independently due to weakness and dysmetria. In addition, he needed assistance in most aspects of the functional evaluation of the activities for daily living. In particular, he showed serious restrictions of delicate actions such as those required for writing a letter. Furthermore, he showed severe dysarthria, and consequently was diagnosed with severe ataxic dysarthria in a speech evaluation. However, signs of dysphagia were not clinically observed, and the patient had normal findings in a video-fluoroscopic swallowing study as well. He complained of diplopia, but there was no restriction of extraocular movement. Left and right horizontal nystagmus was observed in the horizontal gaze, while there was no up and down vertical nystagmus in the upward gaze.

Thiamine levels slightly reduced to $15 \mathrm{nmol} / \mathrm{L} \mathrm{(20-50}$ $\mathrm{nmol} / \mathrm{L}$ ) in a blood test. HbAlC (Hemoglobin A1C) was $5.8 \%$, blood sugar levels two hours after a meal were 208 $\mathrm{mg} / \mathrm{dl}$, and blood sugar levels with an empty stomach increased to $126 \mathrm{mg} / \mathrm{dl}$. There were no other abnormal findings in the blood test. No specific lesion was
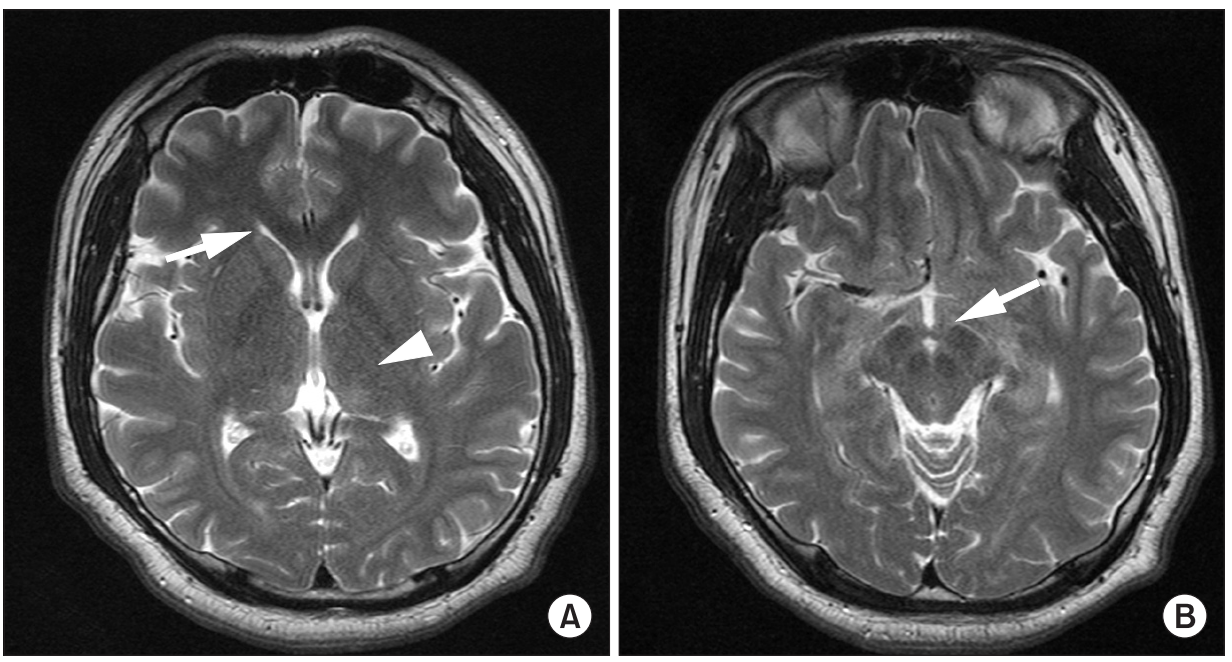

Fig. 1. Brain magnetic resonance imaging (MRI) scans of a 24-yearold man with ataxia, dysarthria, and ophthalmic symptoms. T2-weighted MR images show no definite structural abnormalities or signal changes in the periventricular white matter (arrow), the medial thalamus (arrow head) (A), or the mamillary bodies (arrow) (B). 
observed on magnetic resonance images or angiography that could explain the symptoms (Fig. 1), and the nerve conduction study (NCS), needle electromyography, and somatosensory evoked potentials study were normal.

Although normal findings were seen with neuroimaging and blood tests, the patient showed typical signs of Wernicke's encephalopathy, including ataxia, nystagmus, and diplopia. Furthermore, because he had a medical history of gastrectomy and long-term total parenteral nutrition, we began to administer thiamine immediately. Intravenous $100 \mathrm{mg} /$ day thiamine was administered for 10 days, and then changed to oral administration. One to two days after the start of intravenous administration of thiamine, the patient could stand by himself. One week later, diplopia and nystagmus disappeared, and he could walk a short distance with minimal assistance. After the eighth day of being hospitalized, physical therapy, occupational therapy, and speech therapy were carried out in the department of rehabilitation medicine. As a result, the muscle strength of his limbs and the fine movement of both hands gradually improved. On the tenth day, the muscle strength of the patient's limbs fully recovered to the normal range, and he could do most activities of daily living (ADL) without assistance. On the 30th day after being hospitalized, there remained a slight crawling movement when he walked by himself. Nonetheless, his endurance improved enough to walk more than 20 minutes at a speed of $2.5 \mathrm{~km} /$ hour on a treadmill. In addition, handwriting became almost normal. With continuous speech therapy, ataxic dysarthria also improved to a slight case from a serious case. Finally, he was discharged from the hospital.

\section{DISCUSSION}

Wernicke's encephalopathy usually develops within 6 months after gastrectomy due to thiamine deficiency. Cases have been reported in the past, and most cases were due to the onset of Wernicke's encephalopathy after gastrectomies that were performed for the treatment of a benign or malignant growth in the stomach. ${ }^{5}$ Wernicke's encephalopathy resulting from bariatric surgery for morbid obesity was reported for the first time in $1981,{ }^{3}$ and since then, the number of related reports on it have gradually increased. ${ }^{2}$ There were some cases of Wernicke's encephalopathy after gastrectomy in gastric cancer patients or in patients with diverse chronic diseases that can cause chronic malnutrition. ${ }^{6}$ In Korea, however, there were no reports on Wernicke's encephalopathy after bariatric surgery.

In this case, there were no definite abnormal findings for over four months after sleeve gastrectomy for morbid obesity, but later, the symptoms of typical Wernicke's encephalopathy appeared. Even though he suffered from the symptoms, the patient did not come to the hospital until three months after symptom onset, so when he came to the hospital, he was treated with thiamine administration and rehabilitation.

The typical clinical aspects of Wernicke's encephalopathy are oculomotor disorder, gait disorder, and an altered mental state, but it is very rare for all three of these to appear at the same time, with an incidence of only $16 \% .^{7}$ In addition to these, diplopia, vision perturbations, dysarthria, hypotonia in both legs, and dysphagia may also occur. However, if the clinical symptoms of Wernicke's encephalopathy are doubtful, the diagnosis is usually confirmed when the patients' clinical features are dramatically improved after the administration of thiamine. High signal intensities in the medial thalamus, periventricular white matter, and mamillary bodies are typical findings in T2-weighted magnetic resonance images (MRI) of patients with Wernicke's encephalopathy, but there have been some case reports that have not shown any definite abnormal findings on MRI, similar to this case report. ${ }^{8}$

Thiamine is an essential coenzyme that is involved in glucose metabolism as well as in the movement of acetylcholine and serotonin. If a deficiency of thiamine occurs, it causes a disorder of energy metabolism of brain cells and brings about mobility impairments of neurotransmitters so that neurologic symptoms like those seen in Wernicke's encephalopathy may appear. ${ }^{9}$ The absorption of thiamine, or vitamin B1, is not yet clearly understood but it is known to be absorbed mainly in the duodenum or small intestine (particularly, the ileum and jejunum). Therefore, a deficiency of thiamine is expected to appear with Billroth II anastomosis or gastro-jejunostomy, in which food does not pass through the duodenum. ${ }^{2,10}$ In addition, a deficiency of thiamine may be caused by chronic alcoholism, malnutrition, malignant tumors, or long term intravenous nutrition. ${ }^{10}$ The patient in this report had undergone a laparoscopic sleeve gastrectomy. Since the method did not involve the small intestine, which plays an important role in 
thiamine absorption, the thiamine deficiency may have been caused by the patient's severe vomiting and prolonged parenteral nutrition. In this case, he suffered from a gastro-esophageal anastomosis leakage, and therefore received a high concentration of intravenous nutrition for 60 days without additional supplements of multi-vitamins or micronutrients including thiamine. The deficiency of thiamine generally appears within several weeks after the discontinuation of thiamine, but sometimes it takes several months to develop the deficiency. In Japan, a case was reported in which Wernicke's encephalopathy occurred 28 years after gastrectomy. ${ }^{5}$

If Wernicke's encephalopathy is suspected, immediate administration of thiamine is usually recommended. However, in cases of insufficient administration in the early phase or belated administration, neurological symptoms may unfortunately be sustained even after treatment. ${ }^{4}$ Furthermore, when treatment is inappropriate, serious neurological complications like Korsakov's syndrome may occur, and it is reported that the death rate can reach around $10 \% .^{10}$

Sola et al. ${ }^{4}$ recommended the administration of 1-1.5 $\mathrm{mg} /$ day of thiamine for prophylactic purposes in order to prevent thiamine deficiency after gastrectomy. If a diagnosis of Wernicke's encephalopathy is suspected, it is recommended that the patient be treated with oral administration of thiamine immediately after an administration of thiamine of $50-100 \mathrm{mg} /$ day in an intravenous or intramuscular manner for 1-2 weeks. In this case, the patient did not receive oral administration of thiamine for prophylactic purposes directly after sleeve gastrectomy, but as soon as Wernicke's encephalopathy was suspected, thiamine intravenous injection was given. Finally, it could be confirmed that the patient had Wernicke's encephalopathy when his symptoms quickly improved after the administration. However, mild dysarthria and gait disturbance remained even after the treatment. These symptoms were regarded as sequelae since he did not come to the hospital directly after the symptoms occurred.

Consequently, it is recommended that a patient who has had bariatric surgery take thiamine for prophylactic purposes, even if the patient does not have any symptoms suggesting Wernicke's encephalopathy in the acute phase. It is critical to carefully observe the patient's progress for a considerable period of time after the surgery for the onset of symptoms of Wernicke's encephalopathy. In addition, if Wernicke's encephalopathy occurs, an immediate thiamine injection should be given, and then rehabilitative therapy for the various neurological symptoms is recommended.

\section{REFERENCES}

1. Lanthaler M, Aigner F, Kinzl J, Sieb M, Cakar-Beck F, Nehoda H. Long-term results and complications following adjustable gastric banding. Obes Surg 2010; 20: 1078-1085

2. Makarewicz W, Kaska L, Kobiela J, Stefaniak T, Krajewski J, Stankiewicz M, Wujtewicz MA, Lachinski AJ, Sledzinski Z. Wernicke's syndrome after sleeve gastrectomy. Obes Surg 2007; 17: 704-706

3. Rothrock JF, Smith MS. Wernicke's disease complicating surgical therapy for morbid obesity. J Clin Neuroophthalmol 1981; 1: 195-199

4. Sola E, Morillas C, Garzon S, Ferrer JM, Martin J, Hernandez-Mijares A. Rapid onset of Wernicke's encephalopathy following gastric restrictive surgery. Obes Surg 2003; 13: 661-662

5. Arimura $\mathrm{K}$, Murai $\mathrm{H}$, Kikuchi $\mathrm{H}$, Shigeto $\mathrm{H}$, Taniwaki T, Furuya H, Kira J. Relapsing Wernicke's encephalopathy after gastrectomy. Nippon Naika Gakkai Zasshi 2005; 94: 1606-1608

6. Moon JL, Song DH, Kwon J, Chung YC. Wernicke's encephalopathy caused by dysphagia in down syndrome: a case report. J Korean Acad Rehab Med 2009; 33: $742-745$

7. Sechi G, Serra A. Wernicke's encephalopathy: new clinical settings and recent advances in diagnosis and management. Lancet Neurol 2007; 6: 442-455

8. Zuccoli G, Gallucci M, Capellades J, Regnicolo L, Tumiati B, Giadas TC, Bottari W, Mandrioli J, Bertolini M. Wernicke encephalopathy: MR findings at clinical presentation in twenty-six alcoholic and nonalcoholic patients. AJNR Am J Neuroradiol 2007; 28: 1328-1331

9. Singleton CK, Martin PR. Molecular mechanisms of thiamine utilization. Curr Mol Med 2001; 1: 197-207

10. Chaves LC, Faintuch J, Kahwage S, Alencar Fde A. A cluster of polyneuropathy and Wernicke-Korsakoff syndrome in a bariatric unit. Obes Surg 2002; 12: 328334 